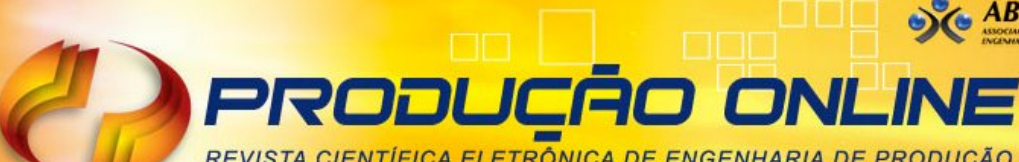 REVISTA CIENTIFICA ELETRÔNICA DE ENGENHARIA DE PRODUÇÃO ISSN 1676-1901
}

\section{FATORES CRÍTICOS DE SUCESSO E CRITÉRIOS DE SUSTENTABILIDADE NA GESTÃO DE MUDANÇAS ORGANIZACIONAIS: UM ESTUDO DE CASO EM UMA EMPRESA DO RAMO QUÍMICO E PETROQUÍMICO}

\section{CRITICAL SUCCESS FACTORS AND SUSTAINABILITY CRITERIA IN THE ORGANIZATIONAL CHANGE MANAGEMENT: A CASE STUDY IN A CHEMICAL AND PETROCHEMINCAL COMPANY}

\author{
Paulo André Souto Mayor Reis* E-mail: psouto@terra.com.br \\ Fernando Gonçalves Amaral * E-mail: amaral@ producao.ufrgs.br \\ *Universidade Federal do Rio Grande do Sul (UFRGS), Porto Alegre, RS
}

Resumo: As mudanças podem ser vistas como situações corriqueiras dentro do processo de gestão de uma organização. Dois elementos são contribuintes como sistemáticas de apoio às mudanças: a Gestão (ou Gerenciamento) das Mudanças (GM) e os Fatores Críticos de Sucesso (FCS) para a mudança, que desejavelmente deveria ser também sustentável. O objetivo do presente artigo consiste em identificar quais FCS são determinantes no processo de Mudança Organizacional (MO), em particular na abordagem da prática de GM de uma empresa química e petroquímica brasileira, associados com a inclusão da gestão proativa da sustentabilidade empresarial, através de Critérios de Sustentabilidade (CS). A metodologia adotada para a pesquisa foi o estudo de caso. Os principais resultados identificaram quarenta e cinco FCS relacionados ao processo de gestão da mudança organizacional e o modelo da Global Report Initiative (GRI) como o mais adotado no meio empresarial mundial. ${ }^{1}$ Foram identificadas contribuições da literatura em FCS no processo de GM vigente na organização, tais como "comunicação constante e eficiente" e "recursos", de forma a incorporar práticas ao modelo atual de gestão da MO. As dimensões de FCS mais relevantes no estudo de caso foram "liderança" e "recursos". Quanto aos CS, foi verificada junto aos respondentes uma visão que enfatiza a dimensão ambiental a despeito da social e econômica, assim como lacunas em ambientação dos mesmos em relação a conceitos de sustentabilidade e sua conexão com FCS na MO.

Palavras-chave: Fatores Críticos de Sucesso. Mudança Organizacional. Sustentabilidade.

Abstract: Changes can be seen as normal situations within the management process of an organization. Two elements are strong contributors as systematic support: management of change (MOC) and the critical success factors (CSF) for change, which also should desirable to be sustainable. The general goal of this work consists in identifying which CSF are determinant in the organizational change process, in specific in the approach of a Brazilian chemical and petrochemical company's organizational MOC process in addition to the inclusion of proactive corporate sustainability management through a present scenario diagnosis. The aim of this paper is to identify which are the decisive CSF in the organizational change process, associated to the proactive management of corporate sustainability, through sustainability criteria. The methodology for the research was the case study. Main results identified forty-five CSF related to the organization's MOC process and the Global Report Initiative (GRI) model as being the most adopted in the world business. Contributions from the literature were identified in CSF in the MOC process within the organization, such as constant and efficient communication process and resources, aiming to incorporate practices to the current organizational change model. Leadership and resources were the most relevant

\footnotetext{
${ }^{1}$ Este texto é resultado de dissertação de mestrado de Paulo André S. M. Reis
} 
dimensions of CSF identified in the case study. Regarding the understanding of sustainability concepts, it was found among the answerers a vision that emphasizes the environmental dimension, despite social and economic dimensions, as well as gaps in their understanding of sustainability concepts and the connection of this subject with CSF in the MOC.

Keywords: Critical Success Factors. Organizational Change. Sustainability.

\section{INTRODUÇÃO}

As transições nas organizações, não importando sua dimensão ou ramo de atuação, passam por processos chamados de Mudanças Organizacionais (MO). Para que sejam atingidos os objetivos desejados, tais mudanças devem ser conduzidas observando-se etapas e critérios, que podem ser entendidos como a Gestão (ou Gerenciamento) de Mudanças (GM). Quanto mais profunda e abrangente a mudança organizacional, maior é a complexidade e o controle necessários e mais detalhado (ou complexo) o seu gerenciamento. No entanto, somente a utilização de uma sistemática de apoio à MO não significa garantia de alcance dos resultados esperados. Soparnot (2011) destaca a necessidade de considerar a MO a partir de uma perspectiva multidimensional e por isso é também importante que se identifique Fatores Críticos de Sucesso (FCS), que contribuirão no desenvolvimento e alcance dos objetivos desta MO.

O relato da sustentabilidade pelas empresas ao redor do mundo tem crescido (KPMG, 2013). Isto é evidenciado pela adoção de diversos modelos, como as normas da série ISO 14000, o índice Dow Jones de Sustentabilidade, o padrão de Responsabilidade Social SA 8000 e as diretrizes de sustentabilidade da Global Report Initiative (GRI) (LOZANO, 2011), como um meio das organizações se tornarem mais sustentáveis e contribuírem para o desenvolvimento sustentável (GRI, 2013), assim como para demonstrarem a quaisquer partes interessadas (stakeholders) o seu alinhamento ao padrão de sustentabilidade reportado.

Destaca-se, assim, uma oportunidade de explorar a prática de GM, considerando a necessidade de visão multidimensional estabelecida por Soparnot (2011), através da inclusão da dimensão da sustentabilidade associada à adoção de FCS, viabilizando assim uma visão de atuação proativa na efetividade do processo de MO e, principalmente, na adoção de Critérios de Sustentabilidade (CS) neste, permitindo que a sustentabilidade em uma organização possa ser efetivamente 
construída ao invés de somente reportada. Adicionalmente, de acordo com Adams e Frost (2008), tem havido supreendentemente pouca pesquisa nos processos de reporte da sustentabilidade e a extensão na qual dados coletados são utilizados no processo decisório dentro das organizações.

\section{FUNDAMENTAÇÃO TEÓRICA}

\subsection{Mudança organizacional}

A mudança - ou a inovação - é uma resposta a um problema (SOUZA, 2010). Planejar a MO envolvendo os diferentes níveis organizacionais e suas atitudes pode ajudar as empresas a melhor superar a resistência à mudança e integrar os seus esforços para a sustentabilidade (LOZANO, 2013). A MO, sob uma ótica de processos, pode ser sistematizada através de etapas consecutivas e dependentes. Capelli e Amaral (2010) trazem exemplos dessas sistematizações, destacando os trabalhos propostos por Kettinger (1997) apud Torres (2002), onde a MO é estruturada nas etapas de visualização, iniciação, diagnóstico, redesenho, reconstrução e avaliação.

Na visão de Kotter (1995), uma mudança organizacional percorre oito etapas: estabelecimento de um senso de urgência, criação de uma coalisão administrativa, desenvolvimento de uma visão estratégica, comunicação da visão da mudança, como investir de empowerment os funcionários para ações abrangentes, realização de conquistas em curto prazo, consolidação de ganhos e produção de mais mudanças e estabelecimento de novos métodos na cultura.

Para efeitos deste artigo, será adotada a definição empregada por Capelli e Amaral (2010), que estabelece que uma MO como qualquer alteração, planejada ou não, dos componentes organizacionais: pessoas, trabalho, estrutura formal, cultura, ou relações entre organização e seu ambiente - que gerem consequências relevantes de natureza positiva ou negativa, para a eficiência, eficácia e sustentabilidade organizacional. 


\section{A GESTÃO DE MUDANÇAS}

A Gestão de Mudanças é um processo para avaliação e controle de modificações em projeto, operação, organização ou atividades em uma instalação, previamente à sua implementação, de forma a ter certeza de que novos perigos não sejam introduzidos e que os riscos associados aos perigos existentes aos funcionários, público ou meio ambiente não sejam aumentados inadvertidamente (CCPS, 2008).

$\mathrm{Na} \mathrm{GM}$, itens como contexto, processo e diferenças individuais tem 0 potencial para influenciar o sucesso da mudança (WALKER et al., 2007). O objetivo final do gerenciamento da mudança é a sustentabilidade da organização no longo prazo. A GM desempenha um papel importante no desenvolvimento da organização porque ela provê estabilidade à organização por estudar mudanças internas e externas (HASHIM, 2013).

\subsection{Fatores críticos de sucesso}

Os Fatores Críticos de Sucesso (FCS) podem ser considerados como um número limitado de fatores cujos resultados - se satisfatórios - irão assegurar um desempenho competitivamente bem sucedido para a organização. FCS podem apoiar o processo de desenvolvimento de estratégia conjunturas específicas, como a análise ambiental, por exemplo, que inclui uma avaliação de climas social, político, econômico e os seus impactos gerais em uma indústria e/ou empresa. A análise dos FCS, especificamente no nível macro e no nível da indústria, auxilia na determinação de ameaças e oportunidades, assim como provê meios de identificar as competências essenciais, recursos e habilidades necessárias para ter sucesso em uma indústria em particular ou situação econômica (LEIDECKER; BRUNO, 1984). A Figura 1 mostra os FCS levantados na revisão bibliográfica e suas dimensões. 
Figura 1 - FCS levantados na revisão bibliográfica e suas dimensões

\begin{tabular}{|c|c|}
\hline Fatores Críticos de Sucesso & Dimensões \\
\hline $\begin{array}{l}\text { Acompanhamento frequente } \\
\text { Comunicação constante e eficiente } \\
\text { Comunicação fácil e contínua } \\
\text { Canais de comunicação estabelecidos da organização }\end{array}$ & Comunicação \\
\hline $\begin{array}{l}\text { Apoio da alta liderança } \\
\text { Compromisso } \\
\text { Fatores externos (cronograma, objetivos, apoio da alta liderança) } \\
\text { Liderança e equipe } \\
\text { Massa critica no staff } \\
\text { Necessidade de endereçar a percepção das pessoas de staff da } \\
\text { habilidade da organização em lidar com a mudança }\end{array}$ & Liderança \\
\hline $\begin{array}{l}\text { Contratações } \\
\text { Relacionados ao gerente de projeto e aos membros da equipe } \\
\text { Administração de pessoal } \\
\text { Alocação bem definida dos papeis e responsabilidades } \\
\text { Disponibilização de recursos em quantidade e qualidade adequados } \\
\text { Interações }\end{array}$ & Recursos \\
\hline $\begin{array}{l}\text { Aspectos sociais e culturais } \\
\text { Cultura, estrutura e processo organizacional } \\
\text { Observar continuamente as lições aprendidas } \\
\text { Mudança cultural } \\
\text { Relacionados à organização }\end{array}$ & Aspectos Culturais \\
\hline $\begin{array}{l}\text { Percepção da preparação da organização em lidar com a mudança } \\
\text { Clima político } \\
\text { Politica e estratégia } \\
\text { Utilidade percebida } \\
\text { Treinamento dos usuários na aplicação }\end{array}$ & $\begin{array}{l}\text { Percepção e } \\
\text { Preparação }\end{array}$ \\
\hline $\begin{array}{l}\text { Gestão ativa e dedicada } \\
\text { Gestão de partes interessadas } \\
\text { Relacionados ao projeto } \\
\text { Gestão de projeto }\end{array}$ & Gestão \\
\hline $\begin{array}{l}\text { Normas governamentais ou do setor } \\
\text { Relacionados ao ambiente exterior (da organização) }\end{array}$ & Regulamentos \\
\hline $\begin{array}{l}\text { Sistemas de autorização do trabalho na empresa } \\
\text { Sistemas de informações do gerenciamento de projetos }\end{array}$ & $\begin{array}{l}\text { Sistemas de } \\
\text { informações }\end{array}$ \\
\hline $\begin{array}{l}\text { Infraestrutura } \\
\text { Condições do mercado }\end{array}$ & Infraestrutura \\
\hline
\end{tabular}

Revista Produção Online, Florianópolis, SC, v.16, n. 1, p. 26-48, jan./mar. 2016. 


\begin{tabular}{|l|c|}
\begin{tabular}{|l|} 
Bancos de dados comerciais (sobre riscos específicos do projeto) \\
Ferramentas e metodologia
\end{tabular} & \\
\hline $\begin{array}{l}\text { Recursos } \\
\text { Recursos humanos existentes }\end{array}$ & Recursos \\
\hline $\begin{array}{l}\text { Objetivos e metas clarificados } \\
\text { Planejamento e análise } \\
\text { Avaliação } \\
\text { Critérios de sucesso (lucro, aceitação do cliente) } \\
\text { Tolerância a risco das partes interessadas }\end{array}$ & $\begin{array}{c}\text { Planejamento, objetivos } \\
\text { e metas }\end{array}$ \\
\hline
\end{tabular}

Fonte: Os Autores (2014)

\subsection{Desenvolvimento sustentável e critérios de sustentabilidade}

O relatório Our Common Future (Nosso Futuro Comum), publicado pelo World Commission on Environment and Development (WCED) em 1987, é tido como um ponto inicial para a maioria das discussões em andamento sobre o conceito de desenvolvimento sustentável (MEBRATU, 1998). A definição conceitual trazida pelo relatório contém dois pontos-chave: o conceito de "necessidades", em particular as necessidades essenciais da pobreza do mundo, para a qual deve ser dada prioridade e a ideia de limitações impostas pelo estado da tecnologia e a organização social na capacidade ambiental de atender necessidades presentes e futuras (MEBRATU, 1998).

De acordo com a ISO 26000 (2010), desenvolvimento sustentável é considerado aquele que satisfaz as necessidades do presente sem comprometer a capacidade das futuras gerações de suprir suas próprias necessidades. Refere-se à integração de objetivos de alta qualidade de vida, saúde e prosperidade com justiça social e manutenção da capacidade da Terra de suportar a vida em toda a sua diversidade. Esses objetivos sociais, econômicos e ambientais são interdependentes e reforçam-se mutuamente. Sustentabilidade pode ser considerada o grau de desenvolvimento sustentável de uma organização (ABNT, 2010).

\section{MODELOS DE REPORTE E INDICADORES DE SUSTENTABILIDADE}

A sustentabilidade corporativa é uma jornada para as empresas, na medida em que buscam continuamente ajustar e melhorar suas atividades internas, estrutura, gestão e como elas mobilizam partes interessadas (incluindo o ambiente) para contribuir mais efetivamente para sociedades sustentáveis (LOZANO, 2013). O reporte da sustentabilidade tornou-se uma prática em crescimento em relação às 
tentativas das empresas em responder às expectativas, pressões e críticas de partes interessadas que desejam ser melhor informadas sobre os impactos sociais e ambientais das atividades dos seus negócios (BOIRAL, 2013).

Teles (2012) refere como razões para integrar a sustentabilidade na prática empresarial a redução de custos proveniente da utilização de métodos de produção mais limpa e inovação, a redução de custos relacionados à saúde e segurança, a redução nos custos com mão-de-obra, fácil acesso a instituições de crédito, associados a riscos menores e reputação da empresa. O compromisso com o desenvolvimento sustentável melhora sua reputação, vantagem no mercado, uma vez que a gestão integrada da cadeia de suprimentos permite o desenvolvimento de relações mais profundas com os clientes e atração de investidores éticos, que excluem as empresas associadas a um desempenho social e ambiental inaceitável.

Concebida em 1999, a Global Report Initiative (GRI) rapidamente tornou-se um dos líderes entre os sistemas voluntários de reporte de sustentabilidade. Ela emergiu na crista do movimento de responsabilidade social corporativa e é um descendente dos movimentos sociais dos anos 70. A GRI introduziu três inovações institucionais: um processo multistakeholder para o desenvolvimento de diretrizes de reporte; institucionalização do processo para a produção de sucessivas gerações de diretrizes e a criação de uma organização para servir como administradora das diretrizes e do processo (GRI, 2013).

A estrutura da GRI visa servir como um modelo amplamente aceito para a elaboração de relatórios sobre o desempenho econômico, ambiental e social de uma organização e foi concebida para ser utilizada por organizações de qualquer porte, setor ou localidade; levando em conta as questões práticas enfrentadas por uma série de organizações, desde pequenas empresas até grupos com operações variadas e geograficamente espalhadas, e inclui o conteúdo geral e o específico por setor, acordados globalmente por vários stakeholders, como aplicáveis na divulgação do desempenho de sustentabilidade da organização (GRI, 2006).

Legendre et al. (2013) afirmam que a adoção das diretrizes da GRI é influenciada pelo tamanho da empresa, lucratividade, cultura de negócio do país e tipo de indústria. Roca e Searcy (2011) apud Teles (2012) avaliaram os indicadores utilizados nos relatórios de sustentabilidade de 94 empresas e identificaram que 31 
destes relatórios incluem indicadores explicitamente identificados como indicadores GRI. As diretrizes GRI possuem o mais amplo escopo e tendem a ser o conjunto de diretrizes mais frequentemente utilizado para reporte da sustentabilidade (LOZANO; HUISINGH, 2011).

O Índice de Sustentabilidade Empresarial (ISE) é uma ferramenta para análise comparativa do desempenho das empresas listadas na BM\&FBOVESPA, sob o aspecto da sustentabilidade corporativa, baseada em eficiência econômica, equilíbrio ambiental, justiça social e governança corporativa. É atualizada a partir de questionários que são distribuídos entre as empresas emissoras das ações de maior liquidez da BM\&FBOVESPA (ISE, 2013). Esta ferramenta possui uma particularidade de ser aplicada apenas no Brasil.

O conceito fundamental do ISE é o Triple Bottom Line, que consiste em três medidas. A primeira é uma medida tradicional das corporações, o lucro. A segunda são as pessoas envolvidas com a companhia, ou seja, as corporações devem ter responsabilidade social. Por fim, a terceira é o planeta e a responsabilidade com o meio ambiente (MABEL, 2011). Além disso, o ISE, a partir de 2012, tornou-se um processo auditado externamente; sendo que essa ação buscou conferir ainda mais credibilidade aos seus procedimentos (ISE, 2013).

O índice Dow Jones de Sustentabilidade, Dow Jones Sustainability Index (DJSI), foi lançado em 1999 como o primeiro conjunto de referências globais de sustentabilidade. O DJSI é globalmente reconhecido por investidores como o padrão líder de referência para sustentabilidade corporativa. O DJSI rastreia o desempenho de companhias líderes mundialmente em critérios econômicos, ambientais e sociais, provendo a investidores referências objetivas para o gerenciamento da sustentabilidade dos seus portfolios de investimento.

A ideia em torno do DJSI é que as práticas de sustentabilidade constituem um elemento potencial para uma criação de valor de longo termo, do qual os acionistas se beneficiarão. Estas práticas ajudam a desenvolver oportunidades e gerenciar riscos econômicos, ambientais e sociais. Os conceitos selecionados para mensurar a Responsabilidade Social Corporativa (RSC) no DJSI são similares àqueles propostos pelas diretrizes mais frequentemente usadas para RSC (GRI, etc.) e estão prontos para serem utilizados por um grande número de empresas para os seus 
relatórios de sustentabilidade. O DJSI introduz um número de indicadores que permite ver o que a organização está fazendo, assim como a avaliação de ativos intangíveis, desenvolvimento de capital humano, questões organizacionais, planos estratégicos, governança corporativa e relações com investidores (LOPEZ et al., 2007).

Identifica-se, de toda forma, que cada um dos modelos que estabelecem uma estrutura para o relato da sustentabilidade possuem seus pontos fortes e fracos. Dentre as diversas abordagens existentes sobre o tema de sustentabilidade, escolheu-se o modelo de reporte de indicadores publicado pela entidade GRI, na sua versão G4 (GRI, 2013), por possuir característica de ser um padrão mais abrangente mundialmente (KPMG, 2013) e também por ser o adotado na empresa objeto do estudo de caso.

\section{PROCEDIMENTOS METODOLÓGICOS}

A problemática enfocada neste artigo aborda a identificação de quais FCS são determinantes no processo de mudança organizacional, em particular na abordagem da prática do GM de uma empresa química e petroquímica brasileira, com operações de primeira e segunda geração no Brasil e exterior, assim como escritórios e bases comerciais em todos continentes, exceto Oceania, incluindo a gestão proativa da sustentabilidade empresarial.

A estrutura metodológica da pesquisa foi constituída das seguintes macro etapas: revisão bibliográfica, seleção dos FCS e CS e desenvolvimento de questionários para levantamento de informações junto aos respondentes. Com esta estrutura estabelecida, viabilizou-se a realização de um estudo de caso, do tipo observação participante, aplicando os questionários elaborados previamente. O estudo de caso é uma investigação empírica que observa um fenômeno contemporâneo em profundidade e em seu contexto de vida real, especialmente quando os limites entre o fenômeno e o contexto não são claramente evidentes (YIN, 1994). Por fim, foi realizada análise dos dados levantados, de modo a viabilizar a discussão e conclusões. 
A pesquisa foi delimitada pelos seguintes fatores: (i) amostra composta por dez respondentes, correspondendo a vinte e cinco por cento do espaço amostral; (ii) área de atuação (engenheiros de diversas áreas e especialidades, por serem estes os profissionais que mais utilizam os procedimentos de gerenciamento de mudanças na organização). Não foi estabelecida nenhuma restrição para as respostas discursivas. As respostas objetivas ficaram restritas a uma opção por questão.

Os respondentes foram selecionados de forma não aleatória, por conveniência, de acordo com os seguintes critérios: representatividade regional onde a organização atua, ambientação no processo de gerenciamento de MO (ser ponto focal ou referência no tema na sua região de atuação ou nacionalmente, participar ativamente no processo), assim como por integrarem equipes multidisciplinares no processo de gestão de mudanças da organização, dentre os diversos papéis particularmente detalhados. Buscando resguardar a identidade dos respondentes, optou-se por referir cada um deles por meio de siglas: "R1" para o primeiro respondente, "R2" para o segundo respondente e assim por diante, até o décimo respondente.

O levantamento do perfil dos respondentes foi realizado através da identificação da área, setor e programa onde atuam, qual o cargo que ocupam, tempo de empresa, formação e o papel preponderante no processo de gestão de mudanças (provoca a mudança, sofre a mudança, é validador/aprovador no processo ou desempenha outro papel).

O estudo de caso realizado visou evidenciar a percepção dos usuários-chave do processo de GM sobre a sistemática adotada atualmente na organização, com foco em FCS e CS criando, por fim, massa crítica de informações que viabilizaram as discussões e conclusões.

Os dados foram coletados através de questionários padronizados, desenvolvidos pelos autores e enviados aos respondentes através de correio eletrônico, durante o estudo de caso. Foram utilizados modelos de questionários de entradas de texto livre para levantamento do perfil dos respondentes e visão geral sobre os temas de gerenciamento de $\mathrm{MO}$ e sustentabilidade, bem como questionários que tinham por fim coletar informações quantitativas para análise 
crítica, dentro dos objetivos referidos para a realização do estudo de caso, respectivamente para FCS e CS.

Os respondentes manifestaram sua percepção sobre os temas de MO e CS através das seguintes questões: "Comente brevemente o que você entende por sustentabilidade.", "Você conhece algum padrão de reporte de sustentabilidade? Caso positivo, qual?", "Comente brevemente se já recebeu alguma informação estruturada sobre a política/visão de sustentabilidade da Organização.", "Você conhece o modo como a empresa divulga seus resultados de sustentabilidade? Caso positivo, descreva.", "Comente como a política de sustentabilidade da empresa influencia em suas atividades rotineiras.", "Especificamente sobre o tema de Mudanças Organizacionais, você percebe conexão dos requisitos do procedimento de Gestão de Mudanças com as dimensões Econômica, Social e Ambiental da Sustentabilidade? Caso positivo, cite exemplos.".

Os itens de apuração da percepção dos respondentes sobre os FCS levantados na revisão da literatura foram: "De modo geral, você considera este fator como de contribuição relevante gerenciamento de mudanças?", "Este fator é considerado no processo de gerenciamento de mudanças vigente na empresa?" e "Escala de importância do Fator Crítico de Sucesso no processo de Gestão de Mudanças, segundo sua percepção".

Para mensurar a conexão dos CS no processo de MO, foi aplicado um questionário cruzando os requisitos do GRI, na sua versão G4, com dois critérios: "grau de aplicação ou aplicabilidade" e "escala de contribuição" . Para cada um deles, foi definida uma escala de Likert com as opções "não aplicável, neutro e aplicável" e "irrelevante, neutro e relevante", respectivamente. Uma parte deste questionário pode ser visto na Figura 2.

Figura 2 - Parte do questionário para apuração da contribuição e importância de CS no processo de $\mathrm{MO}$, com base no padrão GRI - G4.

\begin{tabular}{|c|l|l|l|l|l|l|}
\hline $\begin{array}{c}\text { Indicador } \\
\text { G4 }\end{array}$ & Dimensão & \multicolumn{2}{|c|}{ Requisitos } & \multicolumn{2}{|c|}{$\begin{array}{c}\text { CRITÉRIO 1 } \\
\text { Grau de aplicação ou } \\
\text { aplicabilidade }\end{array}$} & \multicolumn{2}{c|}{$\begin{array}{c}\text { CRITÉRIO 2 } \\
\text { Escala de contribuição } \\
\text { aplicável }\end{array}$} & $\begin{array}{c}\text { não } \\
\text { aplicável }\end{array}$ & neutro & $\begin{array}{c}\text { fortemente } \\
\text { irrelevante }\end{array}$ & neutro & $\begin{array}{c}\text { muito } \\
\text { relevante }\end{array}$ \\
\hline G4-EC1 & Econômico & $\begin{array}{l}\text { É esperado que as mudanças tragam } \\
\text { impactos positivos no resultado } \\
\text { econômico? }\end{array}$ & & & & \\
\hline G4-EC4 & Econômico & $\begin{array}{l}\text { Considera-se assistência financeira } \\
\text { governamental no processo de mudança? }\end{array}$ & & & & \\
\hline
\end{tabular}




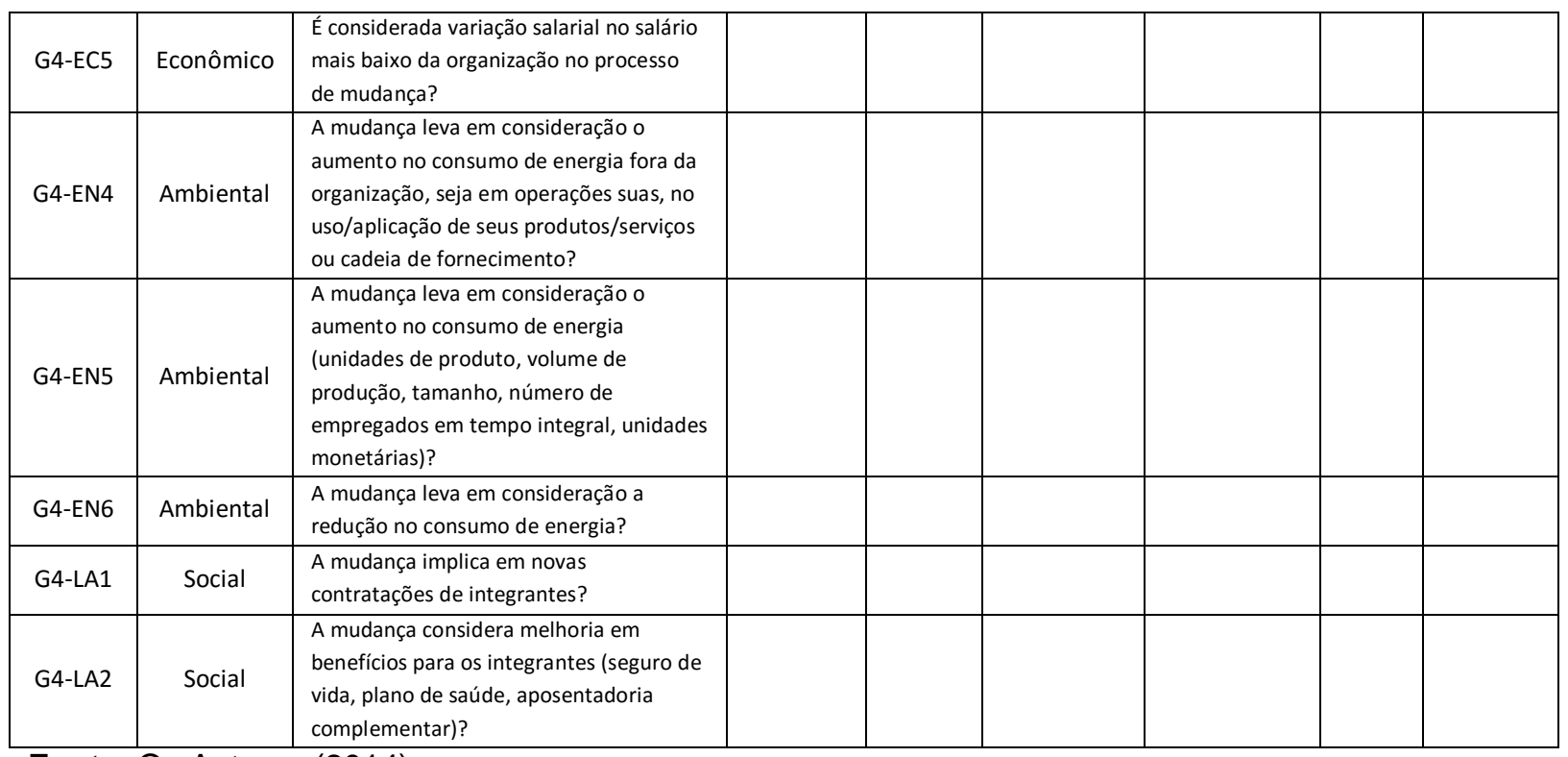

Fonte: Os Autores (2014)

\section{RESULTADOS}

Foi requerido aos autores da pesquisa manter não revelada a identidade da empresa objeto do estudo de caso. Dentre os valores da empresa destaca-se a atuação responsável, em linha com as premissas do desenvolvimento sustentável, reinvestimentos de resultados para a criação de novas oportunidades de trabalho e desenvolvimento da sociedade, assim como retorno aos acionistas e investidores, dentro de um ciclo de desenvolvimento. A organização pratica o gerenciamento de mudanças em sua rotina, como é comum em empresas de grande porte e grau de risco operacional e este processo é estruturado incluindo procedimentos e responsabilidades bem definidas em toda a empresa. Suas etapas são: identificação de uma necessidade de mudança, avaliação preliminar da necessidade de mudança, classificação da mudança, desenho da mudança, avaliação do potencial de risco, análise de risco da mudança, autorização da mudança, implantação da mudança por disciplina, implantação total da mudança, atualização da documentação, treinamento das pessoas afetadas pela mudança, revisão de segurança de pré-partida, autorização de partida, avaliação da eficácia da mudança, avaliação da eficácia do processo de mudança, comunicação ao iniciador da mudança e demais envolvidos e a etapa da conclusão do processo de mudança. 
O levantamento de perfis dos respondentes em relação ao tempo de empresa mostrou que 60\% deles possuem mais de 10 anos de empresa. Em relação à formação acadêmica, 80\% possuem pós-graduação e em relação ao papel de cada um no processo de gerenciamento das $\mathrm{MO}, 42 \%$ provocam a mudança e $33 \%$ são validadores e/ou aprovadores da mudança.

Em relação à percepção da contribuição dos FCS como relevantes no contexto do gerenciamento das MO da organização objeto do estudo de caso, os fatores de maior pontuação indicados pelos respondentes foram: "cultura, estrutura e processo organizacional" (PMI, 2008), "canais de comunicação estabelecidos da organização" (PMI, 2008), "apoio da alta liderança” (CHRUSCIEL; FIELD, 2006), "liderança e equipe" (WESTERVELD, 2003), "percepção da preparação da organização em lidar com a mudança" (CHRUSCIEL; FIELD, 2006), "treinamento dos usuários na aplicação" (CHRUSCIEL; FIELD, 2006), "objetivos e metas clarificados" (CAPELLI; AMARAL, 2010), "planejamento e análise" (CHRUSCIEL; FIELD, 2006) e "disponibilização de recursos em quantidade e qualidade adequados" (CAPELLI; AMARAL, 2010). Depois de agrupadas, as dimensões mais relevantes na observação realizada para este quesito foram "liderança", "recursos", "percepção e preparação", "aspectos culturais" e "planejamento, objetivos e metas".

Para os FCS informados pelos respondentes como considerados no processo de gerenciamento de MO vigente na empresa objeto do estudo de caso, apenas um deles obteve pontuação máxima na indicação dos respondentes: "sistemas de autorização do trabalho na empresa" (PMI, 2008), seguido por: "apoio da alta liderança" (CHRUSCIEL; FIELD, 2006), "normas governamentais ou do setor" (PMI, 2008), "ferramentas e metodologia" (CLARKE et al., 1997), "objetivos e metas clarificados" (CAPELLI; AMARAL, 2010) e "planejamento e análise" (CHRUSCIEL; FIELD, 2006). Depois de agrupadas, as dimensões mais relevantes na observação realizada para este quesito foram "liderança" e "recursos", seguidas por "planejamento, objetivos e metas".

Em relação à gestão da sustentabilidade empresarial, a organização objeto do estudo de caso divulga, desde a sua constituição, seu relatório anual para as partes interessadas internas e externas, adotando as diretrizes GRI para o reporte da sustentabilidade. A metodologia utilizada para a elaboração dos relatórios de 
sustentabilidade é composta por um processo de entrevistas e coletas de dados, através de planilhas eletrônicas, com pessoas dos mais diversos níveis e setores da organização, que são identificadas como pontos focais dos temas reportados nos indicadores. Estas informações, uma vez finalizado o processo de coleta, são analisadas por uma equipe especializada, tratadas e finalmente compiladas na forma final do relatório dos indicadores GRI.

A análise dos dados obtidos no questionário mostrado na Figura 2 viabilizou um agrupamento dos requisitos GRI mais referenciados pelos respondentes, possibilitando por fim um levantamento analítico do percentual de representatividade das dimensões Ambiental, Social e Econômica dentre todas as respostas, mostrando que a maior importância é dada à dimensão ambiental, seguida pelas dimensões social e econômica.

As respostas para a pergunta "O que você entende por Sustentabilidade?" mostraram que as referências de maior frequência estão relacionadas à preservação de recursos, especialmente naturais, de forma a evitar a sua escassez, o que significa uma associação mais representativa à dimensão ambiental quando se aborda o tema da sustentabilidade. Apenas um dos respondentes expressou no seu entendimento a presença das três dimensões (ambiental, social e econômica) da sustentabilidade.

Sobre conhecimento sobre padrões de reporte de sustentabilidade, buscou-se avaliar se os respondentes já tiveram acesso a materiais (relatórios, publicações, comunicados), normas ou padrões, voluntários ou compulsórios, ligados ao tema da sustentabilidade. As respostas mais assertivas referiram relatórios de empresas de grande porte no mercado brasileiro e o padrão GRI como modelo de reporte da sustentabilidade.

Em relação à ambientação dos respondentes na política e visão de sustentabilidade da organização, foi possível concluir que por parte da empresa existem ações de diversas naturezas, em diversos meios e mídias, para comunicar interna e externamente às partes interessadas os seus objetivos, metas e desempenho em sustentabilidade. Apenas um dos respondentes manifestou expressamente conhecer que a organização reporta os indicadores do padrão GRI. Nenhum dos respondentes manifestou ter recebido capacitação formal e/ou 
estruturada em relação a temas, política ou visão de sustentabilidade da organização.

No que diz respeito à influência exercida pela política de sustentabilidade da organização em relação às suas atividades rotineiras, foi constatada uma característica fortemente voltada para a dimensão ambiental: “... acompanhamento dos eco indicadores, através dos quais procuramos otimizar o uso dos recursos naturais auxiliares à produção.”, “... otimização dos nossos processos produtivos para que consumam menos energia e matérias primas.”, “... maior produtividade das plantas com os menores custos (incluindo custo energético)...", "Acompanhamento de eco indicadores.", "Influência pela constante busca de redução do eco indicadores...", "Evolução positiva dos indicadores ambientais (consumo de energia elétrica, efluentes líquidos, resíduos sólidos, água)...", seguida de referências mais pontuais a aspectos genéricos, sobre segurança ocupacional e uma das respostas caracterizando as três dimensões da sustentabilidade.

Quando questionados os respondentes a respeito da conexão entre o tema de MO e as dimensões econômica, social e ambiental da sustentabilidade, as percepções apresentadas mostraram-se bastante dispersas. Dois respondentes manifestaram não haver conexão entre os temas de mudança organizacional/gestão de mudanças e as dimensões ambiental, econômica e social da sustentabilidade. Dois respondentes manifestaram haver conexão através de assuntos de meio ambiente e outros dois sob o aspecto da Segurança de Processos. Apenas um dos respondentes manifestou enxergar uma conexão clara entre os temas e outro manifestou haver conexão, mas afirmou que o tema de sustentabilidade na mudança ainda não é devidamente tratado na organização. A tal cenário pode ser atribuída uma justificativa que reside na falta de conexão e consequente percepção distorcida dos trabalhadores de como as etapas e/ou atividades do processo de gerenciamento de mudanças podem estar ligadas a requisitos de sustentabilidade. Para alguns respondentes que manifestaram com clareza a conexão, é possível que tenham passado por algum tipo de capacitação externa, formal ou informal, bem como possam ter naturalmente mais afinidade com o tema.

De modo geral, entende-se como uma oportunidade da empresa estruturar-se sistematicamente para, tanto capacitar, quanto tornar mais clara a associação dos 
requisitos do procedimento de gestão de mudanças com aqueles da sustentabilidade, especificamente do GRI, no presente caso.

\section{DISCUSSÃO}

\subsection{Fatores críticos de sucesso na gestão de mudanças}

O aspecto de recursos mostra representatividade como FCS na organização em questão. Este aspecto possui uma característica de permear diversos processos dentro da GM da empresa, podendo ser evidenciado desde o planejamento da mudança, passando pelas definições financeiras e estruturais, culturais, de equipe para autorizar, avaliar, executar e revisar a mudança e para que o status pósmudança possa ser mantido, sempre que o requisito de continuidade do negócio se fizer necessário.

A visão que caracteriza a percepção do cenário atual do processo de GM nesta empresa evidencia um estágio de compliance, uma vez que a própria classificação de ocorrência dos FCS referenciados neste quesito permite constatar que elementos básicos de elementos de gestão administrativa estão presentes: (sistema de autorização de trabalho, apoio da alta liderança, normas governamentais ou do setor, ferramentas e metodologia, objetivos e metas, análise). Entenda-se por compliance um estágio onde a organização busca o cumprimento de requisitos administrativos e gerenciais elementares, assim como conformidade legal e voluntária.

As dimensões de maior representatividade para os FCS foram "liderança" e "recursos" (CHRUSCIEL, FIELD, 2006; CLARKE, GARSIDE, 1997; WESTERVELD, 2003). Estas dimensões foram preponderantemente evidenciadas nas três perguntas relacionadas aos FCS no estudo de caso. Neste ponto, destaca-se a importância atribuída para questões, principalmente de natureza administrativa de uma organização e, em particular, a do presente estudo de caso, em particular o apoio e compromisso expressos da alta liderança e o reconhecimento da mudança como parte integral da estratégia de negócio (CLARKE et al., 1997). Os FCS identificados como importantes para a gestão da MO, porém ausentes no processo atual da empresa objeto do estudo de caso é apresentado na Figura 3. 
Figura 3 - FCS identificados como importantes para a gestão da MO, porém ausentes no processo atual da empresa objeto do estudo de caso

\begin{tabular}{|c|l|}
\hline \multirow{2}{*}{ Dimensão } & \multicolumn{1}{|c|}{$\begin{array}{c}\text { FCS indicado como importante para a gestão da Mo, porém } \\
\text { ausente no processo atual }\end{array}$} \\
\hline Aspectos Culturais & Cultura, estrutura e processo organizacional \\
\hline \multirow{2}{*}{ Comunicação } & Canais de comunicação estabelecidos da organização \\
\cline { 2 - 3 } & Comunicação constante e eficiente \\
\hline Gestão & Gestão de projeto \\
\hline Liderança & Compromisso \\
\hline \multirow{2}{*}{ Percepção e Preparação } & Percepção da preparação da organização em lidar com a mudança \\
\cline { 2 - 2 } & Treinamento dos usuários na aplicação \\
\hline \multirow{2}{*}{ Recursos } & Disponibilização de recursos em quantidade e qualidade adequados \\
\cline { 2 - 2 } & Recursos \\
\hline
\end{tabular}

Fonte: Os Autores (2014)

\subsection{Critérios de sustentabilidade na gestão de mudanças}

Em relação ao grau de aplicação ou aplicabilidade para os critérios da GRI, na versão G4, dentro do processo de GM da empresa, destacaram-se por serem mais referenciados pelos respondentes os requisitos "O processo de mudança leva em consideração a redução no consumo de energia" e "O processo de mudança considera impactos nas taxas de frequência de acidentes e doenças", nas dimensões ambiental e social, respectivamente. O agrupamento de ocorrências contabilizou um total de oito requisitos de dimensão ambiental e um de dimensão social.

A partir dos grupos de requisitos mais representativos obtidos através da análise das respostas a esta questão, foi possível determinar que a dimensão mais representativa para os respondentes foi a ambiental (55\%), seguida pela social (39\%) e por último a econômica (6\%). Em alinhamento com a análise ora apresentada, Lozano (2008) identificou em estudo anterior que definições de desenvolvimento sustentável focavam somente na abordagem ambiental, principalmente através do uso de recursos naturais.

Em relação ao grau de contribuição percebida de aplicação ou aplicabilidade para os critérios da GRI na gestão proativa da sustentabilidade e dentro do processo de gerenciamento de $\mathrm{MO}$ da empresa, obteve-se maior ocorrência junto aos 
respondentes o requisito "O processo de mudança considera impactos nas taxas de frequência de acidentes e doenças", pertencente à dimensão social. Os demais requisitos de pontuação significativa são da dimensão ambiental: "variação no consumo de energia", "reciclagem e reuso de água", "aumento da emissão de Gases de Efeito Estufa", "geração ou aquisição de energia", "aumento no volume de descarte de água", "aumento no volume de resíduos", "aumento no volume de vazamentos significativos", "ações de proteção ambiental".

A partir dos grupos de requisitos mais representativos obtidos através da análise das respostas a esta questão, foi possível determinar que a dimensão mais representativa foi a ambiental (47\%), seguida pela social (40\%) e por último a econômica (13\%). Esta avaliação demonstra que a visão de enfoque prioritariamente ambiental ao tema de sustentabilidade dentro desta organização é predominante neste critério avaliado, corroborando a percepção registrada na literatura por Lozano (2008).

Uma das formas de alcançar uma gestão mais eficaz da sustentabilidade é a busca de uma atuação proativa, de forma que um relatório de sustentabilidade seja fruto de uma construção ao longo do ciclo de reporte, ao invés de ser meramente um registro de ações e desempenhos. Dentre as diversas abordagens possíveis, figuram as propostas da inclusão, de forma mais aprofundada, de CS nos processos da empresa, em especial aqueles que tratam da mudança organizacional, assim como a ampliação da divulgação do tema dentro da organização.

Lozano e Huisingh (2011) evidenciam a importância da comunicação com as partes interessadas nas atividades diárias da organização, como uma das estratégias da abordagem da sustentabilidade. Isto deve ocorrer de maneira que os participantes de todos os níveis hierárquicos consigam perceberem-se como atores da construção da gestão da sustentabilidade, a despeito de meros expectadores.

É da mesma forma relevante que seja compreendido que a gestão da sustentabilidade vai além dos critérios ambientais, permeando também os temas sociais e econômicos. Adams e Frost (2011) identificaram que a falta de conhecimento e entendimento do que a sustentabilidade significa para a organização e como a organização se mobiliza em relação às suas prioridades é um fator chave limitante para a integração. Hahn e Kühnen (2013) destacam que a 
literatura atual aparenta estar distante de considerar realmente completo o relato da sustentabilidade nas suas três dimensões.

Entendendo que as mudanças em uma organização sempre ocorrem dentro de um processo específico e de acordo com características próprias, assim como no sentido de fortalecer a atuação proativa da organização em relação ao atendimento aos objetivos da sustentabilidade, destacam-se as seguintes necessidades: melhoria da percepção das pessoas tanto da existência dessa relação como do valor que ela possui para a organização; compreensão das pessoas de forma mais clara sobre a relação entre o que a organização reporta anualmente (neste caso, os indicadores GRI) em relatórios divulgados interna e externamente a partes interessadas, seus requisitos e sua interação com os processos de gestão de mudança; entendimento da conexão das suas atividades com o tema da sustentabilidade e, mais do que isso, como garantir meios de realização de análises críticas e execução das mesmas em prol da gestão proativa da sustentabilidade dentro do contexto da MO.

Adicionalmente, destaca-se a importância de que os atores do processo de MO consigam visualizar com clareza a conexão das dimensões econômica e social ao tema de sustentabilidade (MILLAR et al., 2012).

\section{CONCLUSÃO}

Os resultados obtidos apontaram contribuições da literatura em relação aos FCS atualmente presentes no processo de GM da organização objeto do estudo de caso. Na percepção dos respondentes, o estágio atual identificou a presença de FCS relacionados a temas relacionados a um momento atual de cultura de busca de conformidade na organização, devido à presença de FCS que também podem ser considerados como requisitos administrativos e gerenciais elementares, assim como conformidade legal e voluntária. No que diz respeito à escala de importância, o fator "apoio da alta liderança" foi apontado pelos respondentes como o de maior destaque. De modo mais abrangente, as dimensões "liderança" e "recursos" foram preponderantemente evidenciadas nas três perguntas relacionadas aos FCS no estudo de caso. 
Sobre CS na gestão de MO, o grau de aplicação ou aplicabilidade percebido pelos respondentes para os critérios GRI - versão G4 - mostrou destaque para os requisitos "o processo de mudanças leva em consideração a redução no consumo de energia" e "o processo de mudanças considera impactos nas taxas de frequência de acidentes e doenças", nas dimensões ambiental e social, respectivamente. $\mathrm{Na}$ escala de contribuição percebida de aplicação ou aplicabilidade para os critérios da GRI na gestão proativa da sustentabilidade, o requisito de destaque foi "o processo de mudança considera impactos nas taxas de frequência de acidentes e doenças", pertencente à dimensão social, seguido do requisito "inserção de trabalhadores em atividades/áreas com alta incidência de acidentes/doenças ocupacionais", também de dimensão social. Em ambos os casos, as dimensões mais representativas foram a ambiental, seguida pela social e por último, a econômica.

Os questionamentos que demandaram respostas subjetivas evidenciaram uma percepção preponderante do conceito de sustentabilidade ligado à dimensão ambiental. Apenas um dos respondentes manifestou conhecer que a organização reporta anualmente os indicadores do padrão GRI e nenhum deles manifestou ter recebido capacitação formal em temas, política e visão de sustentabilidade da organização. Quando questionados os respondentes sobre a conexão entre MO e as dimensões ambiental, social e econômica da sustentabilidade, os resultados apresentaram comportamento disperso, indicando falta de uniformidade no entendimento conceitual ou falta da conexão prática entre os assuntos.

Com base nas análises das informações coletadas, foi possível concluir que existe uma ênfase desproporcional dos respondentes na visão de contribuição da dimensão ambiental em relação às dimensões social e econômica da sustentabilidade, assim como uma fragilidade de conceitos e/ou conexão deste contexto com a gestão da MO.

Causas para estas constatações podem estar relacionadas à falta de ambientação com os conceitos de sustentabilidade, levando a uma percepção incompleta, que vislumbra apenas o seu aspecto ambiental e despreza os aspectos econômicos e sociais. Também é possível destacar a falta de pleno conhecimento das pessoas na organização em relação à política, visão e objetivos de sustentabilidade da organização, bem como lacunas em comunicação interna e 
ausência de um modelo estruturado que inclua CS no processo de gestão de MO da empresa, de modo a permitir que a gestão da sustentabilidade seja trabalhada ao longo dos ciclos de mudanças e não apenas reportada como resultado, nos relatórios anuais divulgados ao mercado e partes interessadas. Desenvolvimentos nestes tópicos podem servir de objeto para estudos futuros, complementares a este artigo, assim como a abrangência do estudo de caso aqui realizado para outros ramos de atividade econômica ou padrões de reporte de sustentabilidade empresarial.

\section{REFERÊNCIAS}

ADAMS, C., FROST, G. Integrating sustainability reporting into management practices. Accounting Forum, v. 32, n. 4, p. 288-302, 2008. DOI: 10.1016/j.accfor.2008.05.002

ASSOCIAÇÃO BRASILEIRA DE NORMAS TÉCNICAS. NBR ISO 26000: Diretrizes sobre responsabilidade social. Rio de Janeiro, 2010.

BELASSI, W., TUKEL, O. A new framework for determining critical success/failure factors in projects. International Journal of Project Management. v. 14, n.3, p. 141-151, Elsevier Science Ltd, 1996. DOI: 10.1016/0263-7863(95)00064-X.

BOIRAL, O. Sustainability reports as simulacra? A counter-account of A and A+ GRI reports. Accounting, Auditing \& Accountability Journal, v. 26, n. 7, p. 1036-1071, Emerald Group Publishing Limited, 2013. DOI : 10.1108/AAAJ-04-2012-00998.

BOYNTON, A., ZMUD, R. An Assessment of Critical Success Factors. Sloan Management Review. v. 25, n. 4, p. 17-27, ABI/INFORM Global. 1984.

CAPELLI, A., AMARAL, F.G. Mudança Organizacional e Fatores Críticos de Sucesso: Estudo de Caso no Sistema FIERGS. Revista Produção Online, v. 10, n. 1, p. 71-94, 2010. DOI: $10.14488 / 1676-1901 . v 10 i 1.469$

Center for Chemical Process Safety (CCPS), American Institute of Chemical Engineers (AIChe). Guidelines for Management of Change for Process Safety. New Jersey: John Wiley \& Sons, 2008.

CHRUSCIEL, D., FIELD, D. W. Success factors in dealing with significant change in an organization. Business Process Management Journal, v. 12, n. 4, p. 503-516, 2006. DOI: 10.1108/14637150610678096.

CLARKE, A., GARSIDE, J. The Development of a Best Practice Model For Change Management. European Management Journal, v. 15, n. 5, p. 537-545, 1997. DOI: 10.1016/S0263-2373(97)00033-9. 
Global Reporting Initiative (GRI) - Diretrizes para Relatório de Sustentabilidade, 2006. Disponível em: <https://www.globalreporting.org/resourcelibrary/Brazil-Portuguese-G3Reporting-Guidelines.pdf>. Acesso em: 14 mai. 2013.

GRI. Global Reporting Initiative - What is GRI? Disponível em: $<$ https://www.globalreporting.org/information/about-gri/what-is-

GRI/Pages/default.aspx>. Acesso em: 21 fev. 2012.

Global Reporting Initiative (GRI) - G4 Sustainability Reporting Guidelines - Reporting Principles And Standard Disclosures. Disponível em: $<$ https://www.globalreporting.org/resourcelibrary/GRIG4-Part1-Reporting-Principlesand-Standard-Disclosures.pdf >. Acesso em: 07 out. 2013.

HAHN. R., KÜHNEN, M. Determinants of sustainability reporting: a review of results, trends, theory, and opportunities in an expanding field of research. Journal of Cleaner Production, v. 59, p. 5-21, 2013. DOI: 10.1016/j.jclepro.2013.07.005

HASHIM, M. Change Management. International Journal of Academic Research in Business and Social Sciences, v. 3, n. 7, p. 685-694, 2013. DOI: 10.6007/IJARBSS/v3$\underline{\mathrm{i} 7 / 92}$

ISE. ÍNDICE DE SUSTENTABILIDADE EMPRESARIAL. Disponível em: $<$ http://www.isebvmf.com.br/>. Acesso em: 06 maio 2013.

KOTTER, J. P. Leading Change: Why Transformation Efforts Fail. Harvard Business Review, USA, mar/abr. 1995.

KPMG. KPMG International Survey of Corporate Responsibility Reporting 2013. New York: KPMG.

LEGENDRE, S., CODERRE, F. Determinants of GRI G3 Application Levels: The Case of the Fortune Global 500. Corporate Social Responsibility and Environmental Management, v. 20, n. 3, p. 182-192, 2013. DOI: 10.1002/csr.1285.

LEIDECKER, J. K., BRUNO, A. V., Identifying and Using Critical Success Factors. Long Range Planning, v. 17, n. 1, p. 23-32, 1984. DOI: 10.1016/0024-6301(84)90163-8.

LÓPEZ, M., GARCIA, A, RODRIGUEZ, L. Sustainable Development and Corporate Performance: A Study Based on the Dow Jones Sustainability Index. Journal of Business Ethics, n. 75, p. 285-300, 2007. DOI: 10.1007/s10551-006-9253-8.

LOZANO, R. Are Companies Planning their Organizational Changes for Corporate Sustainability?. Corporate Social Responsibility and Environmental Management, v. 295, n. 5, p. 275-295, 2013. DOI: 10.1002/csr.1290.

LOZANO, R. Envisioning sustainability three-dimensionally. Journal of Cleaner Production, v. 16, n.17, p. 1838-1846, 2008. DOI: 10.1016/j.jclepro.2008.02.008.

LOZANO, R., HUISINGH, D. Inter-linking issues and dimensions in sustainability reporting. Journal of Cleaner Production, v. 19, n.2-3, p. 99-107, 2011. DOI: 10.1016/j.jclepro.2010.01.004. 
MCGRAW HILL FINANCIAL. Dow Jones Sustainability Indices (DJSI) Methodology Disponível em: <http://eu.spindices.com/indices/equity/dow-jones-sustainability-worldindex>. Acesso em: 15 abr. 2014.

MEBRATU, D. Sustainability and sustainable development: historical and conceptual review. Environment Impact Assessment Review, v. 18, p. 493-520, 1998. DOI: 10.1016/S01959255(98)00019-5.

MILLAR, C., HIND, P, MAGALA, S. Sustainability and the need for change: organizational change and transformational vision. Journal of Organizational Change Management, $v$. 25, n. 4, p. 489-500, 2012. DOI: 10.1108/09534811211239272.

Project Management Institute (PMI). GUIA PMBOK - Um Guia do Conhecimento em Gerenciamento de Projetos. Pennsylvania: Project Management Institute, 2008.

SOUZA, B. Gestão da Mudança e da Inovação: arvore de problemas como ferramenta para avaliação do impacto da mudança. Revista de Ciências Gerenciais, v.14, n. 19, p. 89-106, 2010.

SOPARNOT, R. The concept of organizational change capacity. Journal of Organizational Change Management, v. 24, n. 5, p. 640-661, 2011. DOI: 10.1108/09534811111158903.

TELES, C. D. Avaliação monetária da sustentabilidade empresarial. 2012. 190 f. Tese (Doutorado) - Programa de Pós-Graduação em Engenharia de Produção. Universidade Federal do Rio Grande do Sul, Porto Alegre, 2012.

UNITED NATIONS. Our Common Future: Report of the World Commission on Environment and Development. - Disponível em: <http://www.un-documents.net/ocfov.htm>. Acesso em: 10 nov. 2013.

WALKER, H., ARMENAKIN, A., BERNERTH, J. Factors influencing organizational change efforts. Journal of Organizational Change, v. 20, n. 6, p. 761-773, 2007. DOI: 10.1108/09534810710831000.

WESTERVELD, E. The Project Excellence Model: linking success criteria and critical success factors. International Journal of Project Management, v. 21, n. 6, p. 411-418, 2003. DOI: 10.1016/S0263-7863(02)00112-6.

YIN, R. K. Case study research: design and methods. $2^{\text {nd }}$ ed. London: Applied social research methods series, SAGE Publications, v.5, 1994.

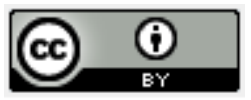

Artigo recebido em 07/08/2014 e aceito para publicação em 08/11/2015

DOI: http://dx.doi.org/ 10.14488/1676-1901.v16i1.1840 